\title{
PREPARATION AND IN VITRO EVALUATION OF LACIDIPINE ORAL LIQUID SOLID TABLET AS AN APPROACH OF SOLUBILITY AND DISSOLUTION RATE ENHANCEMENT
}

\author{
NAWAL A. RAJAB* \\ *Department of Pharmaceutics, College of Pharmacy, University of Baghdad, Iraq \\ Email: dr.nawalrajab@yahoo.com \\ Received: 30 Aug 2017, Revised and Accepted: 28 Nov 2017
}

\begin{abstract}
Objective: The aim of the present study was to prepare a new liquid-solid tablet to enhance the dissolution and bioavailability of a poor water soluble calcium channel blocker lacidipine.

Methods: Firstly, solubility study of lacidipine in different media of water-miscible non-volatile solvents as tween 20, tween 80, propylene glycol, liquid paraffin, PEG200, PEG400, and PEG600 was investigated to select the most suitable solvent. A mathematical model was applied to calculate the appropriate amount of carrier and coating material.

Four liquid-solid tablets of $6 \mathrm{mg}$ lacidipine were prepared by dissolving the drug in the previously chosen water miscible non-volatile solvents, then a binary mixture of the carrier (Avicel PH 102) and coating material (Aerosil 200) at a ratio of 45:1 was used in all preparation since it gave the optimal flow property. Croscarmellose and magnesium stearate were incorporated in all prepared formulas as super disintegrant and lubricant respectively. On the other hand, directly compressed lacidipine tablet of the same previous composition without the addition of any non-volatile solvent was prepared for comparism study. Both characterizations of powder mixture and post-compression tablet evaluations were done. Differential scanning calorimetry (DSC) and fourier transform infrared spectroscopy (FTIR) were investigated for the pure drug, physical mixture, and selected liquid-solid tablet to exclude any drug-excipients interaction.
\end{abstract}

Results: The obtained results indicated that PEG 200 was the most suitable solvent with lacidipine solubility of $2.81 \mathrm{mg} / \mathrm{ml}$. Flowability of all the prepared formulas was found to be within the specification limits. The liquid-solid tablet formula with PEG 200 at $10 \% \mathrm{w} / \mathrm{w}$ lacidipine was the most suitable one in the term of disintegration time ( $21 \pm 0.2$ second), $100 \%$ of drug release within 10 min, and with accepted other tablet properties.

DSC thermograms for both physical mixture of selected liquisolid system and its tablets illustrated the formation of lacidipine amorphous solid solution. The absence of chemical interaction between drug and other formula components was confirmed by remaining all characteristic peaks of lacidipine in all investigated FTIR spectra.

Conclusion: Liquid-solid tablet was considered as a promising system to enhance solubility and dissolution rate of poor-water soluble lacidipine.

Keywords: Lacidipine, Solubility, Liquid-solid system, Dissolution rate

(C) 2018 The Authors. Published by Innovare Academic Sciences Pvt Ltd. This is an open access article under the CC BY license (http://creativecommons.org/licenses/by/4.0/)

DOI: http://dx.doi.org/10.22159/ijap.2018v10i1.22313

\section{INTRODUCTION}

The poor dissolution rate of water-insoluble drugs is still a substantial problem confronting the pharmaceutical industry. A great number of new and possibly, beneficial chemical entities do not reach the public merely because of their poor oral bioavailability due to inadequate dissolution [1].

Due to their low bioavailability, poorly water-soluble drugs cause many difficulties in the development of pharmaceutical dosage forms for oral delivery [2]. Different techniques have been published in the scientific literature to enhance the dissolution profile and also the absorption efficiency and bioavailability of water-insoluble and/or liquid lipophilic drugs.

Reduction of the particle size via micronisation or nanonisation leading to the increased surface area, use of surfactants, lyophilisation, use of cosolvents, self-emulsification and self-microemulsification [2-3].

The most promising and innovative technique for promoting dissolution and in vivo bioavailability of poorly soluble drugs is the formulation of liquisolid systems [4]. Its main advantages include simplicity of manufacturing, use of commercially available excipients, and application of well-known methods and equipment utilized for the manufacturing of conventional tablets [5].

The liquisolid systems are generally considered as acceptably flowing and compressible powdered forms of liquid medications (that implies liquid lipophilic (oily) drugs, or water-insoluble solid drugs dissolved in suitable water-miscible nonvolatile solvent systems [6].
Such liquid medicament may be converted into a dry looking, nonsticky, acceptably flowing, and easily compressible powders by a simple admixture with selected powder excipients referred to as the carrier and coating materials [7]. The technique of liquisolid compacts has been successfully employed to improve the in vitro release of poorly water-soluble drugs such as carbamazepine, famotidine, piroxicam, indomethacin, hydrocortisone, naproxen, furosemide, and prednisolone [8].

Lacidipine (LCDP) is chemically a 1, 4-dihydropyridine derivative which is pharmacologically a calcium channel blocker used as an anti-hypertensive drug. LCDP works by blocking calcium channels in the arterial wall those are present in the muscle cell [9]. The chemical structure of lacidipine was shown in fig. (1)

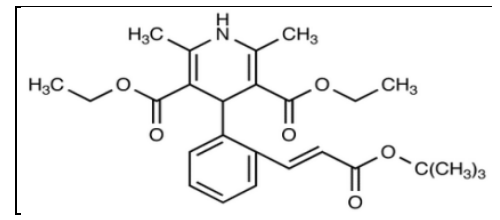

Fig. 1: Chemical structure of lacidipine [10]

In the present study, a poorly soluble calcium channel blocker lacidipine were prepared as liquisolid compacts using water-miscible non-volatile solvents to enhance solubility and the dissolution rate 
with compare the in vitro drug release profile of formulated liquisolid tablets with the prepared direct compressed tablet.

\section{MATERIALS AND METHODS}

\section{Materials}

The following materials were used: Lacidipine was purchased from Hangzhou Hyper Chemical Limited, China., Avicel PH 102 and Aerosil 200 (Wuhan Senwayer Century chemical Co., Ltd), croscarmellose sodium (Rajesh Chemicals, Mumbai; India), Propylene glycols, polyethylene glycol (PEG 200), polyethylene glycol (PEG 400), polyethylene glycol (PEG 600), tween 80, tween 20 and liquid paraffin were obtained from SD Fine Chem Ltd., Mumbai, India. All reagents used were of analytical grade.

\section{Methods}

\section{Solubility studies}

Solubility studies of lacidipine were carried out in water, tween solution, propylene glycol, PEG 200, PEG 400, PEG 600, tween 80, tween 20 and liquid paraffin. Saturated solutions were prepared by adding an excess drug to the vehicles and shaking in a water bath with a shaker for $48 \mathrm{~h}$ at $25 \pm 0.5^{\circ} \mathrm{C}$ under constant vibration. After this period the solutions were filtered, diluted and analyzed by spectrophotometer at $\lambda_{\max } 282 \mathrm{~nm}$ (cary, Australia). Three determinations were carried out for each sample to calculate the solubility of lacidipine.

\section{Application of the mathematical model for designing the liquisolid systems}

A powder can only retain a limited amount of liquid medication while maintaining acceptable flowability and compressibility. Therefore, in order to attain a liquisolid system with acceptable flowable and compressible properties,a mathematical model introduced and validated by Spireas is recommended to calculate the appropriate quantities of carrier and coating material [11].

The model is based on two fundamental properties of a powder, i.e., flowable liquid retention potential ( $\Phi$ value) and compressible liquid retention potential ( $\Psi$ value). The $\Phi$ and $\Psi$ values of a powder excipient represent the maximum quantity of liquid vehicle that can be retained in the powder bulk without compromising flowability and compressibility [12].

In this study, PEG 200, Microcrystalline Cellulose (Avicel PH 102MCC), and Aerosil 200 were used as a liquid vehicle, carrier, coating respectively. The concentration of the drug in the liquid vehicle was varied and the carrier: coating ratio was kept constant in all formulations $(\mathrm{R}=45: 1)$.
Depending on the excipient ratio $(R)$ of the powder substrate an acceptably flowing and compressible liquisolid system can be obtained only if a maximum liquid load on the carrier material is not exceeded. This liquid/carrier ratio is termed "liquid load factor $L_{f}$ $[\mathrm{w} / \mathrm{w}]$ and is defined as the weight ratio of the liquid formulation $(W)$ and the carrier material $(Q)$ in the system:

$$
\mathrm{L}_{\mathrm{f}}=\mathrm{W} \div \mathrm{Q}------(1)
$$

$R$ represents the ratio between the weights of the carrier $(Q)$ and the coating $(q)$ material present in the formulation:

$$
\mathrm{R}=\mathrm{Q} \div \mathrm{q}------(2)
$$

The liquid load factor that ensures acceptable flowability $\left(\mathrm{L}_{\mathrm{f}}\right)$ can be determined by:

$$
\mathrm{Lf}=\Phi+\varphi \cdot(1 / \mathrm{R})----(3)
$$

Where $\Phi$ and $\varphi$ are the $\Phi$-values of the carrier and coating material, respectively [13].

In order to calculate the required ingredient quantities, the flowable liquid retention potentials ( $\Phi$-values) of powder excipients were utilized. In polyethene glycol 200, the $\Phi$-value of Avicel PH 102 was found to be 0.007 and the $\varphi$-value for Aerosil 200 was 3.26 .

So, by knowing both $\mathrm{L}_{\mathrm{f}}$ and $\mathrm{W}$, the appropriate quantities of carrier $(\mathrm{Q})$ and coating (q) powder materials required to convert a given amount of liquid medication (W) into an acceptably flowing and compressible liquisolid system could be calculated from equation (1) and (2).

\section{Preparation of lacidipine liquisolid compact}

Four liquisolid tablets denoted (F1 to F4) containing 6 mg of lacidipine were prepared by dispersing in the non-volatile vehicle, by choosing a non-volatile solvent for dissolving the drug. From the results of solubility studies, PEG 200 is chosen as the liquid vehicle due to higher solubility profile of lacidipine in it. Then a bindery mixture of the carrier (Avicel PH 102) and coating material (Aerosil 200) was prepared at a ratio of 45: 1 , by continuous mixing for a period of 10 min in a mortar. The amount of carrier and coating materials are enough to maintain acceptable flow and compression properties. R 45 was used in all formulations since it gave the optimal flow property.

Finally, a $5 \% \mathrm{w} / \mathrm{w}$ of croscarmellose as a super disintegrant was added and mixed for $10 \mathrm{~min}$ then $1 \% \mathrm{w} / \mathrm{w}$ of magnesium stearate as a lubricant was added into the mixture and mixed for $2 \mathrm{~min}$. The final mixture was compacted using a single punch-tablet machine (Korsch EKO, Germany). The composition of liquisolid tablets was

\begin{tabular}{|c|c|c|c|c|c|c|c|c|c|c|}
\hline Formula & $\begin{array}{l}\text { Lacidipine } \\
\text { concentration In } \\
\text { liquid medication } \\
(\% w / w)\end{array}$ & $\begin{array}{l}\text { (R) } \\
\text { Q: } \\
\text { q }\end{array}$ & $\begin{array}{l}\text { Loading } \\
\text { factor } \\
\text { (Lf) } \\
\text { W/Q }\end{array}$ & $\begin{array}{l}\text { PEG } \\
200 \\
\text { (mg) }\end{array}$ & $\begin{array}{l}\text { Lacidipine } \\
\text { (mg) }\end{array}$ & $\begin{array}{l}\text { Avicel } \\
\text { PH 102 } \\
\text { Q (mg) }\end{array}$ & $\begin{array}{l}\text { Aerosil } \\
200 \\
\text { q (mg) }\end{array}$ & $\begin{array}{l}\text { Crosscarmelose } \\
5 \%(\mathrm{mg})\end{array}$ & $\begin{array}{l}\text { Magnesium } \\
\text { stearate 1\% } \\
(\mathrm{mg})\end{array}$ & $\begin{array}{l}\text { Unit } \\
\text { dose } \\
\text { (mg) }\end{array}$ \\
\hline F1 & 10 & 45 & 0.079 & 54 & 6 & 759.5 & 17 & 42 & 8.78 & 887 \\
\hline $\mathrm{F} 2$ & 15 & 45 & 0.079 & 34 & 6 & 506 & 11.2 & 28 & 5.8 & 591 \\
\hline F3 & 20 & 45 & 0.079 & 24 & 6 & 380 & 8.4 & 21 & 4.4 & 443.8 \\
\hline $\mathrm{F} 4$ & 25 & 45 & 0.079 & 18 & 6 & 304 & 6.8 & 16.7 & 3.5 & 355 \\
\hline
\end{tabular}
shown in the table (1).

Table 1: Formulation of lacidipine liquisolid tablets prepared using PEG-200

\section{Preparation of directly compressed tablets (DCT)}

Compressed tablet containing $6 \mathrm{mg}$ of lacidipine was prepared with direct compression method without addition of any non-volatile liquid vehicle. The lacidipine powder was mixed with suitable amounts of considered carrier and coating material. Afterwards, 5\% of crosscarmelose was added as a disintegrant and mixed, and then $1 \%$ of lubricant was added to the mixture. The final blend was compressed using Korsch (Germany) tablet machine [14].

\section{Pre-compression studies}

\section{Characterization of powder mixture}

\section{Angle of repose}

The frictional force in a loose powder can be measured by the angle of repose. The angle of Repose is the maximum angle between the surface of a pile of powder and horizontal plane. It is usually determined by Fixed funnel Method.

A funnel was secured with its tip at a given height (h), above a Petri dish is placed on a flat horizontal surface. The blend carefully pored through the funnel until the apex of the conical pile just touches the tip of the funnel. The radius (r) of the base of the conical pile was measured.

The angle of repose $(\theta)$ was calculated using the following formula:

$$
\operatorname{Tan} \theta=h \div r
$$

Where; $\theta=$ Angle of repose

$\mathrm{h}=$ Height of the cone in $\mathrm{cm}$

$\mathrm{r}=$ Radius of the cone base in $\mathrm{cm}$ 


\section{The compressibility index (Carr's Index)}

Carr's Index is a measure of the propensity of a powder to be compressed. It is determined from the bulk and tapped densities and is calculated using the following formula [15].

$$
\text { Carr's index }=\frac{\text { Tappeddensity-Bulk density }}{\text { Tappeddensity }} \times 100
$$

Bulk density $=\frac{w}{v o}$

Taped density $=\frac{w}{v f}$

Where $w$ is weight of powder

$\mathrm{V}_{\mathrm{o}}$ is volume of powder

$V_{f}$ is taped volume of powder

\section{Post-compression studies}

\section{Hardness}

The hardness of the tablet was determined using Monsanto hardness tester. Three tablets were randomly selected from each formulation and hardness of the same was determined [16]. The average value was calculated.

\section{Friability testing}

Friability of the tablets was determined by using Roche friabilator. Ten tablets from each batch were placed in the friabilator and rotated at $25 \mathrm{rpm}$ for a period of $4 \mathrm{~min}$. The friability was determined using the following formula.

$$
\text { Percentage friability }=\frac{\mathrm{W} 1-\mathrm{W} 2}{\mathrm{~W} 1} \mathrm{x} 100
$$

Where the W1=Initial weight of 20 tablets, W2=Weight of the 20 tablets after testing [17].

\section{Weight variation}

Twenty tablets were taken and their weight was determined individually and collectively on a digital weighting balance. The average weight of one tablet was determined from the collective weight and comparing the individual tablet weight to average weight variation tolerance [18].

\section{Drug content}

Ten tablets were crushed in a mortar and the powder equivalent to $6 \mathrm{mg}$ of lacidipine was weighed and dissolved in $10 \mathrm{ml}$ of methanol in a $100 \mathrm{ml}$ volumetric flask and the volume was made with $1 \%$ tween 20 solutions. This solution was further diluted with $1 \%$ tween 20 if necessary and the absorbance was measured at $282 \mathrm{~nm}[19]$

\section{Disintegration test}

The disintegration time was determined in water maintained at $37 \pm 2$ C The disintegration apparatus with a basket rack assembly containing six open-ended tubes and 10-mesh screen on the bottom was used. A tablet was placed in each tube of the basket and the time for complete disintegration of the six tablets was recorded [20-22]

\section{In vitro drug dissolution test}

The dissolution rates of all formulations were measured by using tablet dissolution apparatus USP Type II. Dissolution studies were carried out using $500 \mathrm{ml}$ of purified water with $1 \% \mathrm{w} / \mathrm{v}$ polysorbate 20 , at $50 \mathrm{rpm}$ and at temperature of $37 \pm 0.5 \mathrm{C}^{\circ} .10 \mathrm{ml}$ aliq uots were withdrawn at suitable time interval $(5,10,15,20,25,30,35,40$ and $45 \mathrm{~min}$ ), filtered through syringe filter $0.45 \mu \mathrm{m}$ and diluted as per need and replaced with fresh medium.

Sink conditions were maintained throughout the study. The samples were then analyzed at $284 \mathrm{~nm}$ by UV/visible spectrophotometer. The study was carried out in triplicate [23].

\section{Comparison of dissolution rate}

The model-independent approach was applied for comparison of dissolution profiles. For the comparison of dissolution data for each formulation, percentages of drug dissolved at $10 \mathrm{~min}$ (Q10 $\mathrm{min}$ ), $45 \mathrm{~min}$ (Q45 $\mathrm{min}$ ), mean dissolution time (MDT) and percentage of dissolution efficiency (DE) at the end of $45 \mathrm{~min}$ were calculated using DD solver software in order to select the optimized formula.

The dissolution profile of the optimized formula and the DCT was compared on the basis of their similarity factor ( $f 2$ ) and dissimilarity factor (f1). These parameters were determined for tablets from the optimal formulation of liquisolid compact and direct conventional tablet of lacidipine.

Dissimilarity factor was calculated using Equation (1) and similarity factor was calculated using Equation (2).

$$
\mathrm{f} 1=\frac{\sum_{\mathrm{t}}^{\mathrm{n}}=1|\mathrm{Rt}-\mathrm{Tt}|}{\sum_{\mathrm{t}}^{\mathrm{n}}=1 \mathrm{Rt}} \times 100 \text {----------Equation } 1
$$

Where $\mathrm{n}$ is the sampling number, $\mathrm{Rj}$ and $\mathrm{Tj}$ are the percents dissolved of the reference and test products at each time point $\mathrm{j}$. The $f 1$ value between 0 and 15 [24].

$$
\left.f_{2}=50 \log \left\{\left[1+\frac{1}{\mathrm{n}} \sum_{t=1}^{n}\left(R_{t}-T_{t}\right)\right)\right]-0.5 \times 100\right\} \text {----------Equation }
$$

Where $\mathrm{n}$ is the number of time points at which \% dissolved was determined, $\mathrm{Rt}$ is the \% dissolved of one formulation at a given time point, and $\mathrm{Tt}$ is the \% dissolved of the formulation to be compared at the same time point. The similarity factor fits the result between 0 and 100 . It is 100 when the two dissolution profiles are identical and approaches 0 as the dissimilarity increases. An $f 2$ above 50 indicates that the two profiles are similar [25].

\section{Drug-polymer interaction studies \\ Differential scanning calorimetry (DSC)}

Samples (3-5 mg) were placed in an aluminum pan and heated in the DSC-60 (Shimadzu, Japan) at a constant rate of $10{ }^{\circ} \mathrm{C} / \mathrm{min}$, in an atmosphere of nitrogen over a temperature range of 25-300 ${ }^{\circ} \mathrm{C}$. The DSC studies were performed on the pure drug, a physical mixture of liquisolid system and on the selected liquisolid tablet [26].

\section{Fourier-transform infrared spectroscopy (FTIR)}

It was performed using the Infrared spectrophotometer (Lambda 7600 , Australia). Samples of 2-3 mg were mixed with about $100 \mathrm{mg}$ of dry potassium bromide powder and compressed into transparent discs then scanned over a wave range of $4000-400 \mathrm{~cm}^{-1}$ in FTIR instrument. The IR spectra were performed on the pure drug, a physical mixture of liquisolid system and on a selected liquisolid tablet [27]

\section{Statistical analysis}

All the results were expressed as the mean value \pm standard deviation (SD). One way analysis of variance (ANOVA) was used to test for significance, at a $5 \%$ significance level. Statistical difference dealing $(\mathrm{P}<0.05)$ was considered significant [28].

\section{RESULTS AND DISCUSSION}

\section{Saturation solubility studies}

The solubility of lacidipine in different media is presented in table 2 and fig. 2. Drug solubility in a non-volatile vehicle is the most important aspect in liquisolid systems. The solubility of the drug contributes to molecular dispersion in a non-volatile solvent which will improve the dissolution rate. 
Table 2: Solubility of lacidipine in different media

\begin{tabular}{ll}
\hline Medium & Solubility $(\mathbf{m g} / \mathbf{m l})$ \\
\hline Water & $0.044 \pm 0.0035$ \\
Tween solution & $0.09 \pm 0.006$ \\
Tween 20 & $1.413 \pm 0.0635$ \\
Tween 80 & $5.486 \pm 0.0104$ \\
Propylene glycole & $1.4883 \pm 0.01$ \\
Liquid paraffin & $0.823 \pm 0.123$ \\
PEG 200 & $2.81 \pm 0.2$ \\
PEG 400 & $2.523 \pm 0.05$ \\
PEG 600 & $2.654 \pm 0.0582$ \\
\hline
\end{tabular}

(Reading Represent the mean \pm SD, $n=3$ )

From the solubility profile, it can be judged that the drug was soluble in most the non-polar liquid vehicles and very slightly soluble in water. And amongst the non-polar liquid vehicles the drug has the highest solubility in PEG 200 and tween 80, but due to its viscosity, tween 80 was not chosen to prepare the liqisolid. Increasing the moisture content of carrier materials may result in decreased powder flowability [29]. So, PEG 200 was the appropriate solvent in the preparation of lacidipine liquisolid tablets.

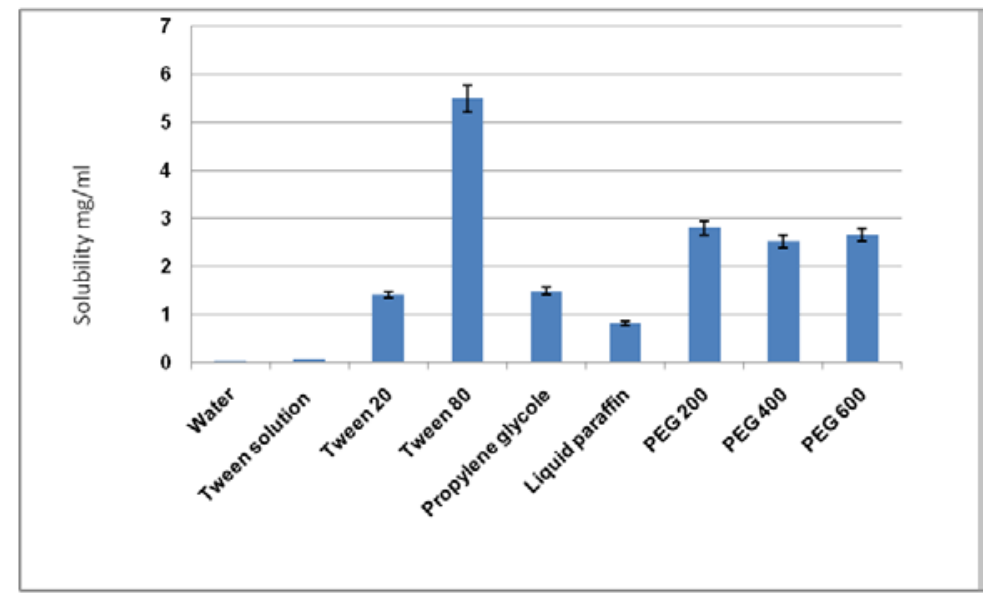

Fig. 2: Drug solubility profile of lacidipine, (reading represent the mean $\pm S D, n=3$ )

\section{Flowable liquid retention potential ( $\Phi$ value) and liquid load factor (Lf)}

$\Phi$ value of Carrier and Coat materials in polyethene glycol 200 were cited in the literature and found to be 0.007 and 3.26 respectively. According to mathematical model proposed by Spireas et al. equation for Avicel PH-102 and Aerosil 200 in polyethene glycol 200 was calculated by using $R$ value $=45$ as

$$
\mathbf{L f}=0.007+3.26(1 / R)
$$

So, $\mathrm{f}=0.079$

\section{Precompression evaluation}

\section{Angle of repose}

Flowability of a powder is of critical importance in the formulation and industrial production of tablet dosage form. As a general guide, powders with angles of repose greater than 50 have poor flow properties, whereas minimum angles close to 25 correspond to very good flow properties [30].
The results of the angle of repose and carr's index were given in the table 3 . The results showed that angle of repose was ranged from $33.5^{\circ}$ to $36.3^{\circ}$ for the formulated liquisolid powder, while the angle of repose for DCT was 25meaning excellent flowability due to the absence of the liquid vehicle. It was found that formula of highest drug concentration in PEG 200 has better flowability than another formula due to fewer amounts of vehicle presents. And the results of carr's index were proved all formulations shown flow property ranged from fair to poor.

From table 3, it can be concluded that all the formulas were found to be within the specification limits.

\section{Hardness test}

The hardness of the prepared tablets of all formulations is within the acceptable limit. The hardness of tablets prepared by direct compression was found to be from 6 to $10 \mathrm{~kg} / \mathrm{cm}^{2}$ as shown in table 4 . Generally, the ideal tablet hardness should be produced without applying excessive compression force where rapid tablet disintegration and drug dissolution are maintained at the same time [31].

Table 3: Flow properties of lacidipine liquisolid powder

\begin{tabular}{llll}
\hline Formula & Angle of repose & Type of flow & Carr's index \\
\hline F1 & $36 \pm 1.1$ & Fair & $21.8 \pm 2.4$ \\
F2 & $36 \pm 1.6$ & Fair & $23 \pm 2.8$ \\
F3 & $34 \pm 2.5$ & Good & $21.3 \pm 1.5$ \\
F4 & $33.5 \pm 3.1$ & Good & $20 \pm 2.5$ \\
DCT & $25 \pm 2.1$ & Excellent & $15 \pm 1$ \\
\hline
\end{tabular}

(Reading Represent the mean $\pm S D, n=3$ ) 


\section{Friability test}

All lacidipine tablets had acceptable friability as none of the tested formulas exceeded 1\% loss in tablet weight as shown in table 4; also, no tablet was cracked, split or broken in either formulation. Since all the prepared formulas met the standard friability criteria, they are expected to show acceptable durability and withstand abrasion.

\section{Disintegration time}

The disintegration time for the prepared lacidipine liquisolid tablets was shown in table 4. It was found that, the mean of the disintegration times for all investigated tablets was less than $1 \mathrm{~min}$, due to the effect of superdisintegrants, microcrystalline cellulose and croscarmellose accelerate the disintegration of the liquisolid compact and as the drug concentration in liquid medication increases, and the disintegration time is increased [32].

\section{Weight variation}

Tablets of each formula were subjected to weight variation test, the difference in weight and percent deviation was calculated for each tablet. The results of the test as demonstrated in table 4 showed that, the tablet weights were within the pharmacoepial limit.

\section{Drug content}

The results indicate that the contents for tablets of all the formulations were uniform and contains a therapeutic dose of the active ingredients as shown in table 4 .

Table 4: Evaluation of different parameters of liquisolid tablet and DCT

\begin{tabular}{llllll}
\hline Formula & $\begin{array}{l}\text { Hardness } \\
\left(\mathbf{K g} / \mathbf{c m}^{\mathbf{2}}\right)\end{array}$ & $\begin{array}{l}\text { Friability } \\
\mathbf{( \% )}\end{array}$ & Disintegration time (s) & $\begin{array}{l}\text { Content uniformity } \\
(\mathbf{\%})\end{array}$ & $\begin{array}{l}\text { Weight variation } \\
(\mathbf{m g})\end{array}$ \\
\hline F1 & $10 \pm 0.6$ & 0.18 & $21 \pm 0.2$ & $80 \pm 0.4$ & $88 \pm 0.3$ \\
F2 & $9 \pm 0.3$ & 0.4 & $13 \pm 0.6$ & $100 \pm 0.2$ & $589.8 \pm 2.7$ \\
F3 & $6 \pm 0.3$ & 0.77 & $25 \pm 0.7$ & $85 \pm 0.2$ & \\
F4 & $6 \pm 0.3$ & 0.14 & $39 \pm 0.2$ & $100 \pm 0.1$ \\
DCT & $10 \pm 1.6$ & 0.4 & $23 \pm 0.2$ & $352.7 \pm 1.6$ \\
\hline
\end{tabular}

(Values represent the mean $\pm \mathrm{SD}, \mathrm{n}=3$ )

\section{In vitro drug dissolution studies}

The results of in vitro drug released at different time intervals is plotted against time to obtain the dissolution profiles as shown in fig. 3 and 4 .
Liquisolid formulations initially show greater release than DCT. MDT, Q10 min, Q45 min and DE of each liquisolid formula and DCT were calculated in tween 20 solutions and reported in table 5 .

Table 5: Dissolution parameters of liquisolid compacts and DCT

\begin{tabular}{llll}
\hline Formula & Q10 min & Q45 min & MDT (min) \\
\hline F1 & $100 \pm 0.9$ & $98 \pm 0.3$ & 3.83 \\
F2 & $55 \pm 1.2$ & $100 \pm 0.6$ & 11.6 \\
F3 & $83 \pm 2$ & $100 \pm 1$ & 6.95 \\
F4 & $58 \pm 0.5$ & $100 \pm 1$ & 10.35 \\
DCT & $48 \pm 0.7$ & $90 \pm 0.4$ & 12.72 \\
\hline
\end{tabular}

(Reading represent the mean $\pm S D, n=3$ )

The results in the table clearly affirm that the liquisolid tablet F1 had the highest percentage $100 \%$ of lacidipine dissolved in $10 \mathrm{~min}$, while $48 \%$ of the drug was released from DCT. All liquisolid tablets show completely $100 \%$ of drug release at $45 \mathrm{~min}$. However, DCT show less drug release at this time.

DE is commonly applied for comparison of dissolution profiles to decide better formulation. DE of F1 was found to be $90 \%$. Higher DE indicated that liquisolid compact has significantly enhanced dissolution rate $(p<0.05)$. From the result, it is concluded that concentration of drug in liquid medication is an important factor in drug release [33]. A decrease in concentration of drug in PEG 200 increases the dispersion of drug at molecular level which may further enhance the dissolution rate of the drug [34].

Lower MDT values indicated faster release of drug from liquisolid formulation, MDT of DCT was found to be $12.72 \mathrm{~min}$ while that of formulation FA3 was 3.83 minute. Hence the formula (F1) was considered as the optimized tablet.

Pairwise procedure such as dissimilarity (f1) and similarity (f2) factors provides a simple way to compare dissolution data. FDA guidance proposes that $f 1$ value between 0 and 15 and $f 2$ value between50-100 indicate equivalence in dissolution profiles.

In comparison between optimized tablet $\mathrm{F} 1$ and DCT, $f 1$ value was $36.74>15$ and $f 2$ values were $26.43<50$ indicate nonequivalence in dissolution profile between F1 and DCT. Such enhanced drug dissolution rate may be mainly attributed to the fact that this poorly- water-soluble drug is already in solution in PEG 200, while at the same time, it is carried by the powder particles (microcrystalline cellulose-silica) of the liquisolid vehicle. Thus, its release is accelerated due to its markedly increased wetability and surface availability to the dissolution medium [35].

\section{Differential scanning calorimetry (DSC)}

The DSC thermogram of the drug in fig. 5 depicts a sharp endothermic peak at $186{ }^{\circ} \mathrm{C}$ corresponding to the melting temperature of lacidipine. Such sharp endothermic peak signifies that drug used was in the pure crystalline state [36]. A complete disappearance of the drug melting peak was observed in the physical mixture of optimized liquisolid system F1 and its tablet as shown in fig. 6 and 7 respectively, a fact that agrees with the formation of drug solution in the liquisolid powdered system, i.e., the drug was molecularly dispersed within the liquisolid matrix and also indicate the formation of an amorphous solid solution [37].

\section{Fourier-transform infrared spectroscopy (FTIR)}

The FTIR spectrum of lacidipine, physical mixture and liquisolid tablet of F1 was illustrated in fig. 8, 9 and 10 respectively. The characteristic absorption peaks of lacidipine appeared at $3348.78,2978.52$ to 2808 , 1702 to $1653.66,1629.55$ and $1292 \mathrm{~cm}^{-1}$, respectively, denoting stretching vibration of- $\mathrm{NH}$ of dihydropyridine ring, $-\mathrm{CH}-,-\mathrm{C}=\mathrm{O},-\mathrm{C}=\mathrm{C}$ functional groups and ester group[[38]]. The optimized liquisolid system showed characteristic peaks of lacidipine and carriers. These results indicated that there is no chemical interaction between drug 
and carrier when formed as a liquisolid system. As shown in fig. 9 and 10 , there is a reduction in intensity of the characteristic absorption bands of drug in liquisolid formulations which might be attributed to the hydrogen bonding interaction of the amin group of lacidipine with the hydroxyl group of the PEG 200. This resulted in drug dissolution enhancement as shown by dissolution data [34].

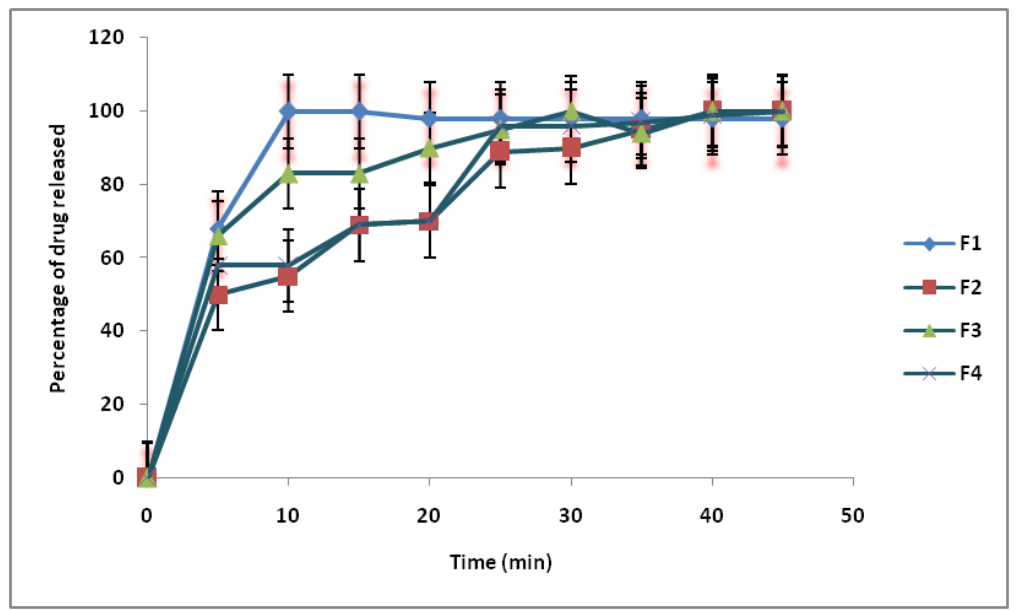

Fig. 3: Dissolution profile of lacidipine from liquisolid compact (Data expressed as a mean $\pm S D, n=3$ )

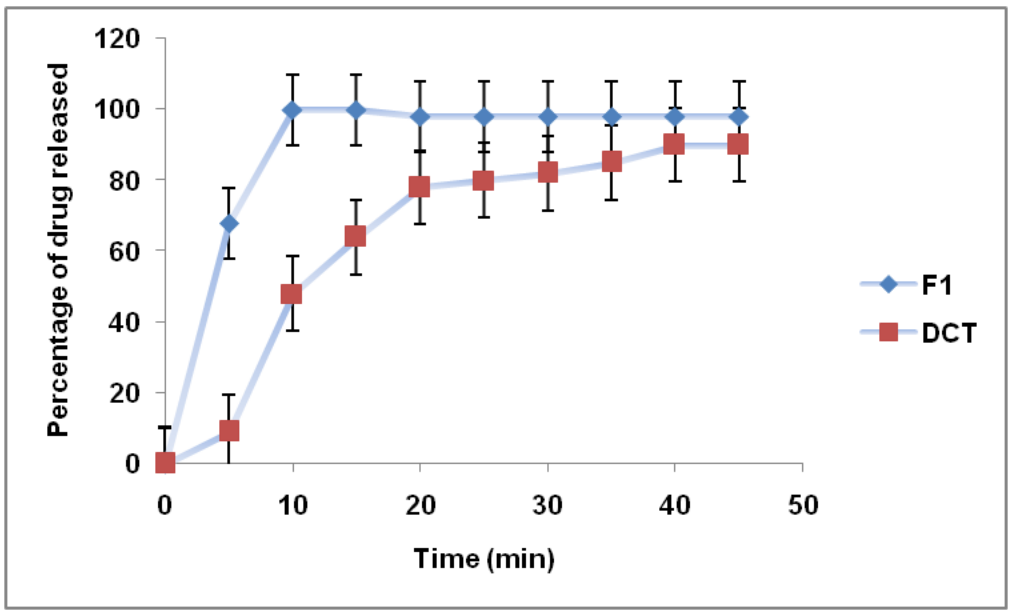

Fig. 4: Dissolution profile of lacidipine from F1 and direct conventional tablet, (data expressed as a mean \pm SD, $n=3$ )

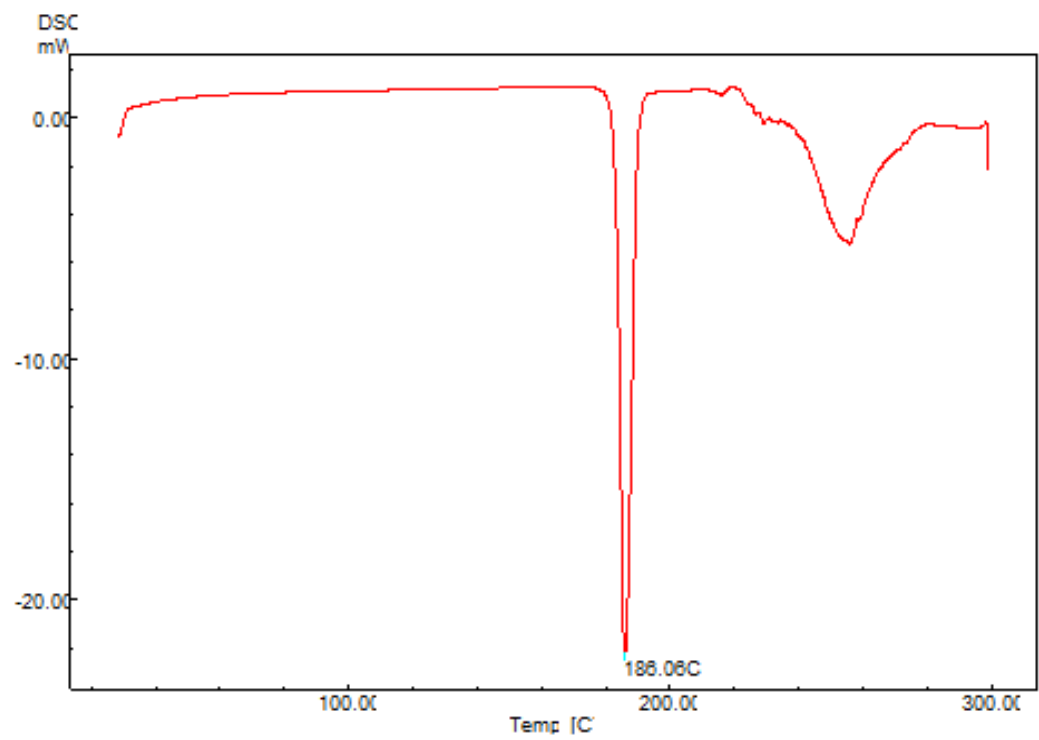

Fig. 5: DSC thermogram of lacidipine 


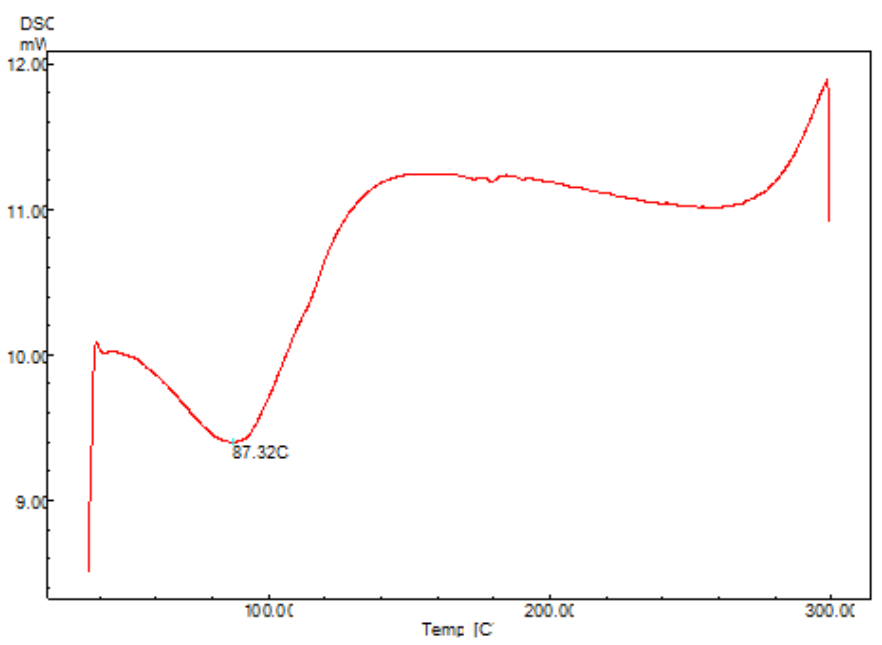

Fig. 6: DSC thermogram of a physical mixture of F1 liquisolid system

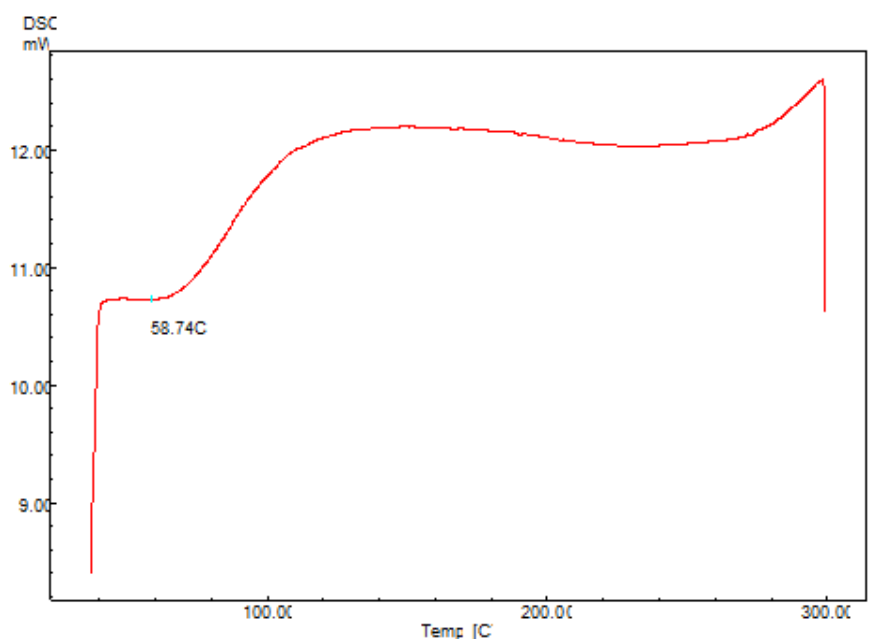

Fig. 7: DSC thermogram of F1 liquisolid tablet

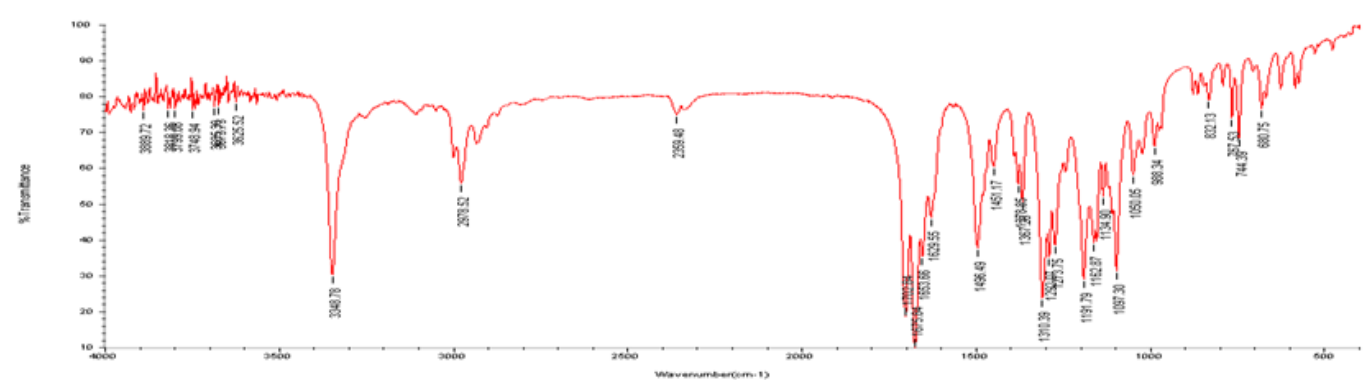

Fig. 8: FTIR spectrum of lacidipine

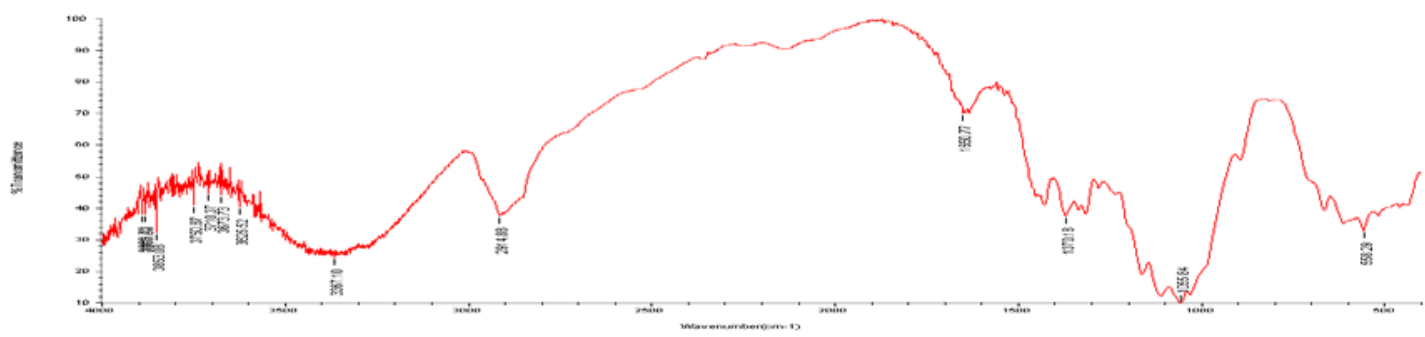

Fig. 9: FTIR spectrum of a physical mixture of F1 liquisolid system 


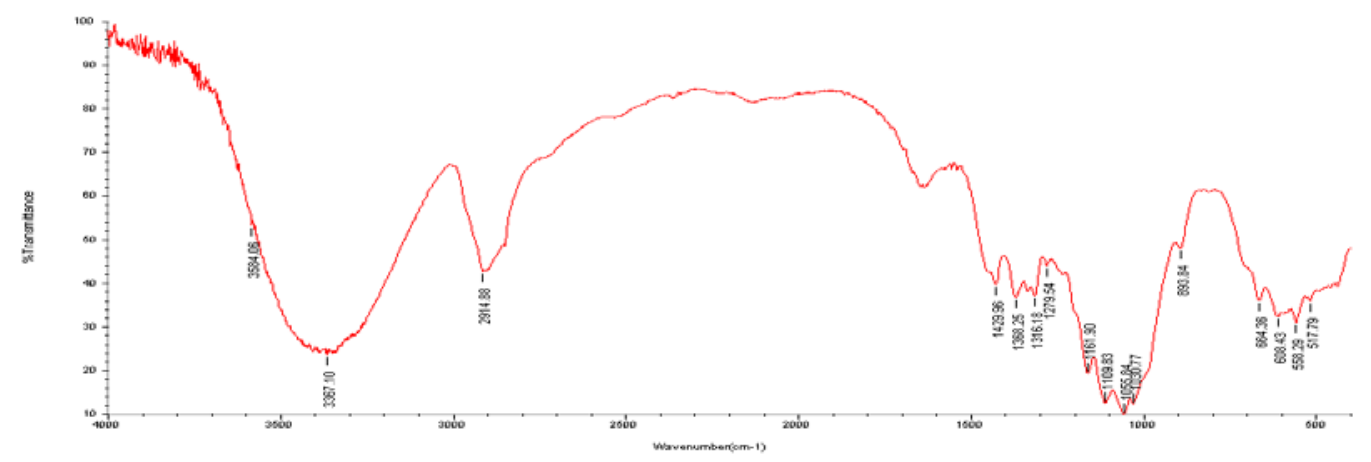

Fig. 10: FTIR spectrum of F1 liquisolid tablet

\section{CONCLUSION}

The overall objective of present study was to enhance dissolution of poorly water-soluble lacidipine by liquisolid compact technique. The liquisolid tablets formulated with the PEG 200 at a drug concentration of $10 \% \mathrm{w} / \mathrm{w}$ is the best formulation among all the batches of liquisolid tablets prepared, in terms of faster disintegration time, superior dissolution profile, and acceptable tablet properties. PEG 200 was found to be a promising liquid vehicle in formulating liquisolid formulations of lacidipine. The liquid vehicle plays a contributing role in improving the dissolution profiles of a poorly water-soluble drug in the liquisolid formulations.

\section{ACKNOWLEDGEMENT}

I am very thankful to Pharmaceutical department, Collage of Pharmacy, University of Baghdad, Iraq; for providing necessary supporting and facilities for complete this study.

\section{AUTHOR CONTRIBUTION}

All the work carried out by me.

\section{CONFLICTS OF INTERESTS}

Declare none

\section{REFERENCES}

1. Satyajit Panda, R Varaprasad, K Priyanka, Ranjit P Swain. Liquisolid technique: a novel approach for dosage form design. Int J Appl Pharm 2017;9:8-14.

2. Vraníková B, Gajdziok J. Liquisolid systems and aspects influencing their research and development. Acta Pharm 2013;63:447-65.

3. Burra S, Galipelly Sk. Enhancement of solubility and dissolution rate of frusemide through liquisolid technique. Pharm Lett 2010;2:321-8

4. Kumar Nagabandi V, Ramarao T, Jayaveera K. Liquisolid compacts: a novel approach to enhance bioavailability of poorly soluble drugs. Int J Pharm Biol Sci 2011;1:89-102.

5. Kurek M, Woyna-Orlewicz K, Khalid Mh, Jachowicz R. Optimization of furosemide liquisolid tablets preparation process leading to their mass and size reduction. Acta Poloniae Pharm Drug Res 2016;73:1325-31.

6. Patel Bb, Shah Cn. Recent research on liquisolid technology for solubility enhancement-a review. Int J Adv Pharm 2016;5:1-7.

7. Asija R, Bhatt S, Asija S, Yadav A, Shah I. Enhancement of solubility and dissolution of lercanidipine by liquisolid technique. J Chem Pharm Res 2014;6:2680-6.

8. Nagabandi V, Tadikonda R, Jayaveera K. Formulation development and evaluation of liquisolid systems to improve the dissolution rate of ketoprofen. Int J Biomed Res 2011;2:530-41.

9. Patel Mzr, Patel Mhk, Trivedi Mhj, Patel Dkn, Nayak Dbs. Enhancement of solubility and dissolution properties of lacidipine by solid dispersion. Int J Pharm Drug Anal 2015;3:165-70.

10. Darekar T, Aithal K, Shirodkar R, Kumar L, Attari Z, Lewis S. Characterization and in vivo evaluation of lacidipine inclusion complexes with $\beta$-cyclodextrin and its derivatives. J Inclusion Phenom Macrocyclic Chem 2016;84:225-35.

11. Spireas S. Liquisolid systems and methods of preparing same. Google Patents; 2002.

12. Lu M, Xing H, Jiang J, Chen X, Yang T, Wang D, et al. Liquisolid technique and its applications in pharmaceutics. Asian J Pharm Clin Res 2017;12:115-23.

13. El-Hammadi M, Awad N. Investigating the use of liquisolid compacts technique to minimize the influence of ph variations on loratadine release. AAPS PharmSciTech 2012;13:53-8.

14. Mohammed Asif Hussain Mg, T Rama Rao, Maimuna Anjum. Preparation and evaluation of nilvadipine liquisolid compacts. Int J Pharm Pharm Sci 2014;6:1-8.

15. Vemula S, Radhika K. Liquisolid compact technique for improvement of the dissolution rate of flurbiprofen: formulation and evaluation. J Drug Res Dev 2015;1:1-5.

16. Bhairav Ba, Jadhav Ms, Saudagar R. Formulation and evaluation of liquisolid tablet of felodipine. World J Pharm Pharm Sci 2016;5:70-1685.

17. Mahendran Baskaran Kbt, Gowthamarajan Kuppusamy, Veera, Kollipara Vsrkar. Formulation development of atorvastatin calcium tablets by gel liquisolid compact technique for improving solidstate stability and dissolution profile. J Drug Res Devel 2016;2:1-7.

18. Shahtalebi Ma, Tabbakhian M, Koosha S. Formulation and evaluation of orally disintegrating tablet of ondansetron using natural super disintegrant. J Herb Med Pharmacol 2015;4:102-9.

19. Nl Prasanthi Nrr, Ss Manikiran. Studies on dissolution enhancement of poorly water-soluble drug using water-soluble carriers. Asian J Pharm Clin Res 2010;3:95-7.

20. Altememy D, Altememy JJ. Formulation and evaluation of meloxicam liquisolid compact. Int J Pharm Pharm Sci 2014;6:453-63.

21. Trustees Uspcbo, Convention Usp, Revision Uspcco, Board Nf, Editors. The United States Pharmacopeia: National Formulary. Supplement United States Pharmacopeial Convention; 2006.

22. Deshmukh P. Dissolution enhancement of rosuvastatin calcium by liquisolid compact technique. J Pharm 2013:1-9. http://dx.doi.org/10.1155/2013/315902

23. Amit Mukharya Sc, Niyaz Mansuri, Arun K Misra. Solid-state characterization of lacidipine/Pvp K 29/32 solid dispersion primed by solvent co-evaporation. Int J Pharm Invest 2012;2:90-6.

24. Eedara Bb, Kankane M, Jukanti R, Nagabandi Vk, Bandari S. Enhanced solubility and permeability of exemestane solid dispersion powders for improved oral delivery. Int J Pharm Invest 2013;43:229-42.

25. Adibkia K, Shokri J, Barzegar-Jalali M, Solduzian M, Javadzadeh Y. Effect of solvent type on retardation properties of diltiazem Hcl form liquisolid tablets. Colloids Surf B 2014;113:10-4.

26. Abd-El Bary A, Louis D, Sayed S. Liquisolid tablet formulation as a tool to improve the dissolution of olmesartan medoxomil. Inventi Rapid: Ndds 2014;3:1-8.

27. Rajesh Asija Sb, Sangeeta Asija, Alpesh Yadav, Isha Shah Enhancement of solubility and dissolution of lercanidipine by liquisolid technique. J Chem Pharm Res 2014;6:2680-6.

28. Elkhodairy $\mathrm{Ka}$, Hassan $\mathrm{Ma}$, Afifi Sa. Formulation and optimization of orodispersible tablets of flutamide. Saudi Pharm J 2014;22:53-61. 
29. Chella N, Shastri N, Tadikonda Rr. Use of the liquisolid compact technique for improvement of the dissolution rate of valsartan. Acta Pharm Sin B 2012;2:502-8.

30. Martin A. Physical Pharmacy: Physical Chemical Principles In The Pharmaceutical Sciences: Bi Waverly. Pvt Ltd; 1993.

31. Singh Y. Martin's physical pharmacy and pharmaceutical sciences: department of pharmaceutics ernest mario school of pharmacy rutgers, The State University Of New Jersey; 2006.

32. Sayyad Fj, Tulsankar Sl, Kolap Ub. Design and development of liquisolid compact of candesartan cilexetil to enhance dissolution. J Pharm Res 2013;7:381-8.

33. J Ramesh Bvk, Y Narasimha Reddy. Formulation and evaluation of olanzapine liquisolid compact tablets. Int J Pharm Technol 2016;8:11780-9.

34. Dias $\mathrm{Rj}$, Mali Kk, Ghorpade Vs, Daulatrao V, Havaldar Vrm. Formulation and evaluation of carbamazepine liquisolid compacts using novel carriers. Indian J Pharm Educ Res 2017;51:69-78.

35. Saeedi M, Akbari J, Morteza-Semnani K, Enayati-Fard R, Sar Reshteh-Dar S, Soleymani A. Enhancement of dissolution rate of indomethacin: using liquisolid compacts. Iran J Pharm Res 2011;10:25-34

36. Patel Mzr, Patel Mhk, Trivedi Mhj, Patel Dkn, Nayak Dbs. Enhancement of solubility and dissolution properties of lacidipine by solid dispersion. Int J Pharm Drug Anal 2015;3:165-70.

37. Fahmy Rh, Kassem Ma. Enhancement of famotidine dissolution rate through liquisolid tablets formulation: in vitro and in vivo evaluation. Eur J Pharm Biopharm 2008;69:993-1003.

38. Mukharya A, Chaudhary S, Mansuri N, Misra Ak. Solid-state characterization of lacidipine/Pvp K 29/32 solid dispersion primed by solvent co-evaporation. Int $\mathrm{J}$ Pharm Invest 2012;2:90-6. 